\title{
ORIGINAL
}

\section{ATENCION PRIMARIA Y RESPONSABILIDADES DE SALUD PÚBLICA EN SEIS PAÍSES DE EUROPA Y AMÉRICA DEL NORTE: UN ESTUDIO PILOTO *}

Barbara Starfield (1), Francisco Sevilla (2), Denise Aube (3), Pierre Bergeron (4), Jan M. De Maeseneer (5), Per Hjortdahl (6), John R. Lumpkin (7), José Martínez Olmos (8) y Antonio SarriaSantamera (9)

(1) Johns Hopkins School of Public Health. USA

(2) Equipo Atención Primaria: Pozuelo 1. Madrid. España

(3) National Public Health Institute of Quebec and Laval University. Canadá

(4) National Public Health Institute of Quebec and Laval University. Canadá

(5) Department of General Practice and Primary Health Care. Ghent University. Belgium

(6) Family and Community Medicine. University of Oslo. Norway

(7) Robert Wood Johnson Foundation. USA

(8) Escuela Andaluza de Salud Pública. Granada. España

(9) Agencia Española de Evaluación de Tecnologías. Instituto de Salud Carlos III. España

\section{RESUMEN}

Fundamento: Los rápidos cambios en los sistemas sanitario son una oportunidad para reorientar las relaciones entre sus diferentes componentes. Con el objetivo de conocer dónde se ubica la responsabilidad para la realización de diferentes tipos de actividades preventivas, se realizó una encuesta en ocho áreas geográficas de seis países de Europa y Norteamérica.

Métodos: Encuesta entre expertos basada en una matriz que relaciona servicios sanitarios preventivos con la población a la que se dirigen. Se establecieron ocho situaciones clínicas (vacunaciones infantiles y antigripal adultos; y detección precoz: de cáncer mediante mamografí, de tuberculosis, de Hipertensión Arterial, de fenilcesis), con el fin de conocer en relación a las mismas la población diana, nivel de establecimiento de las políticas, nivel de contacto con los individuos, seguimiento de los individuos con diagnósticos positivos y registro de sus datos clínicos.

Resultados: Este estudio piloto mostró muy escasa coincidencia de los resultados tanto entre las ocho áreas encuestadas como en cada una de ellas. No se encontró un patrón regular para las actividades de prevención exploradas entre los diferentes países, ni en función de la tipología de sus sistemas sanitarios, ni por la orientación hacia la atención primaria de los diferentes sistemas.

Conclusiones: Existe un escaso consenso en las áreas estudiadas en relación con la mejor forma de realizar las intervenciones de salud pública que conllevan prestación de atención sanitaria personal.

Palabras clave: Atención primaria de salud. Prevención. Hospial. Salud Pública. Organizaciones de gestión de servicios. Inmunizaciones. Hipertensión arterial. VIH. Tuberculosis. Gestión.

Correspondencia:

Barbara Starfield

Johns Hopkins School of Public Health

624 North Broadway, Room 452

Baltimore, MD 21205 - USA

Correo electrónico bstarfie@jhsph.edu

\section{ABSTRACT}

\section{Primary Health Care and Public Health} Responsibilities in Six European and North American Countries: A Pilot Study

Background: Rapidly occurring changes within the health care systems are creating an opportunity to re-orient the relationships between their different sectors. In order to know the locus of responsibility for various types of preventive activities, we undertook an inquiry on eight areas in six countries from Europe and North America

Methods: An inquiry among experts based on a matrix which arrayed the type of preventive health services against the targe population. Eight clinical conditions were identified (childhood immunizations; adult influenza vaccination; mammography screening, tuberculosis screening, hypertension screening, PKU screening, HIV screening, and osteoporosis testing) trying to know their target population and the locus of responsibility for setting of policy, target population a policy, f people with abnormal tests and maintenance of their medical records.

Results: This pilot study showed very little results coincidence either within the eight surveyed areas or across them. There was no regular pattern for the preventive activities studied among the different countries, neither according to the type of health system, nor to the primary health care orientation of the different systems.

Conclusions: There was a limited consensus in the activities studied concerning the best mode of doing public health intervention for personal health services.

Key words: Primary Health Care. Preventive Services. Hospital. Public Health. Health Maintenance organizations. Immunizations. Hypetension. VIH. Tuberculosis. Management.

*) Este trabajo ha sido financiado parcialmente por el Grant No. 6 U30 CS 00189-05 S1 R1 del Bureau of Primary Health Care, Health Resources and Services Administration, Department of Health and Human Services, al Primary Care Policy Center for the Underserved en la Johns Hopkins University. 


\section{INTRODUCCIÓN}

La separación entre las funciones de salud pública y las de atención sanitaria es una práctica común en la mayoría de los sistemas sanitarios, incluyendo aquéllos que tienen mayor integración entre los diferentes niveles, como pueden ser los casos del Reino Unido y España. Esta separación afecta incluso a servicios de atención sanitaria individual, como las vacunaciones o la detección precoz de enfermedades que con frecuencia se han considerado patrimonio de la salud pública.

En España, de manera relacionada con los diferentes ritmos en el proceso de descentralización de las competencias sanitarias de los últimos veinte años ${ }^{1}$, ha habido muchas Comunidades Autónomas con competencias plenas en salud pública sin tener transferidas las de atención sanitaria. Este hecho ha provocado que la separación de funciones señalada haya sido en ocasiones muy llamativa, a pesar del proceso de reforma de la Atención Primaria iniciado en 1984 y de lo dispuesto en la Ley General de Sanidad de 1986, por la que se creó el Sistema Nacional de Salud y orientó la actuación de los Servicios de Salud que lo conforman a la protección de la salud, la prevención de la enfermedad y la atención integral.

España ha completado su proceso de transferencias sanitarias en el año 2002, y todas las Comunidades Autónomas tienen ahora plenas competencias para la organización y gestión de sus servicios de salud en un escenario que debe buscar la cooperación entre las administraciones para contribuir a la mejor protección posible del derecho a la salud de todos ${ }^{2}$. Por tanto, parecía un momento apropiado para conocer en tres áreas concretas de España, con competencias diversas hasta ese momento, diferentes aspectos de la relación entre las actividades consideradas de salud pública y las de atención sanitaria, así como evaluar los mismos aspectos en otros países, con el fin de tratar de identificar las formas más habituales de la prestación de dichos servicios en orden a su posible utilidad para aquellas Comunidades Autónomas en proceso de reorganización o adaptación de sus estructuras.

Es bien conocido que los rápidos cambios que están ocurriendo en los sistemas sanitarios, no solo en España, están creando una oportunidad para evaluar y reorientar las relaciones entre los diferentes niveles que los conforman. Entre estos cambios, la integración de las distintas actividades relacionadas con la protección de la salud están condicionando que tanto la salud pública como los servicios sanitarios, especialmente la atención primaria, se muevan hacia nuevas formas de interrelación.

La ausencia de una definición ampliamente aceptada de cada campo hace complicado delimitar los papeles relativos de la salud pública y de la atención primaria. El tratado de Oxford de Salud Pública ${ }^{3}$ resume varios intentos de definir la salud pública; el más sólido parece estar basado en Winslow quien, en 1920, dijo: La Salud Pública es la ciencia y el arte de prevenir la enfermedad, prolongar la vida y promover la salud física a través de los esfuerzos organizados de la sociedad para la seguridad del entorno, el control de las enfermedades infecciosas, la educación de las personas en los principios de la higiene, la organización de los servicios médicos y de enfermería para el diagnóstico precoz y la prevención de la enfermedad, y el desarrollo de la maquinaria social que asegure a cada individuo de la comunidad un estándar de vida adecuado para el mantenimiento de la salud. En 1.988, el Government Committee of Inquiry del Reino Unido adoptó la primera parte de la definición (la salud pública es la ciencia y el arte de prevenir la enfermedad, prolongar la vida y promover la salud física a través de los esfuerzos organizados de la sociedad) y en 1988 el comité IOM en EEUU de Norteamérica (EEUU) la modificó en el siguiente sentido: la salud pública se refiere a los 
esfuerzos organizados de la comunidad dirigidos a la prevención de la enfermedad y la promoción de la salud 4 . Por otro lado, las definiciones de Atención Primaria no son tan consistentes en su orientación a los individuos versus la colectividad, aunque de hecho la Organización Mundial de la Salud, a través de la definición de Alma Ata ${ }^{5}$, claramente asumió una aproximación colectiva y, dada la amplitud de dicha definición, es difícil diferenciar la atención primaria de la salud pública.

Los países con un sistema sanitario basado mayoritariamente en el sector privado se han asociado tradicionalmente con una separación inestable de responsabilidades entre el personal de los servicios sanitarios y el personal de los servicios de salud pública. Sin embargo, el Informe sobre el Futuro de la Salud Pública del Institute of Medicine de $E U^{4}$ señalaba que el sector público en ese país ha asumido la responsabilidad de muchos aspectos de los servicios sanitarios, tanto los relacionados con la prevención y la promoción de la salud (siempre, al menos teóricamente, parte de la atención sanitaria convencional) así como de los servicios sanitarios curativos para poblaciones incapaces de acceder al sector privado.

En España hasta 1984 también existía una clara separación entre las responsabilidades consideradas de salud pública y la atención sanitaria $^{6}$. La reforma iniciada aquel año, inspirada en los postulados planteados en Alma Ata por la Organización Mundial de la Salud, creaba los Equipos de Atención Primaria y les encomendaba la atención integral de los ciudadanos de su Zona Básica de Salud, de manera que con independencia de la administración responsable última de la prestación, éstos recibiesen en su Centro de Salud, o coordinada por los profesionales del mismo, toda la atención en materia de prevención, asistencia o rehabilitación ${ }^{7}$.

En el Reino Unido, los Primary Care Trusts han asumido la responsabilidad de desarrollar y planificar la atención primaria y los servicios comunitarios en su área, mejorar la calidad de sus servicios y la responsabilidad de los servicios hospitalarios que reciben los ciudadanos, pero también la de mejorar la salud de las poblaciones y disminuir las desigualdades en salud ${ }^{8}$.

En países como EEUU, Canadá, Noruega y Bélgica, los servicios de Atención Primaria pueden tener una base poblacional pero, a diferencia de España o Reino Unido, no es lo habitual y cuando lo hacen es en base a la captación de individuos o grupos de individuos en lugar de hacerlo por áreas geográficas.

El objetivo de este estudio piloto es conocer algunas de las interrelaciones entre salud pública y servicios de atención sanitaria individual, e identificar a los responsables de ejecutar diferentes funciones consideradas necesarias para el desarrollo de diversas actividades preventivas en los sistemas sanitarios en seis países de Europa y América del Norte.

\section{MATERIAL Y MÉTODOS}

El estudio realizado es de carácter transversal y descriptivo basado en un cuestionario respondido por expertos, que no busca, en esta fase de carácter piloto, resultados con significación estadística, sino aproximarse a la realidad de cada lugar desde el conocimiento aportado por las personas seleccionadas en cada área estudiada.

La base de la investigación fue una matriz previamente publicada ${ }^{9}$ que relaciona el tipo de servicio sanitario: prevención primaria, prevención secundaria (búsqueda activa y detección de individuos asintomáticos), o prevención terciaria (manejo clínico en presencia de síntomas de un diagnóstico); con la población diana a la que se dirige cada servicio. 
El cuestionario final utilizado por los expertos fue el resultado de un proceso iterativo entre los autores del trabajo que permitió alcanzar un consenso tanto sobre las actividades preventivas a incluir en el estudio como en las cuestiones a investigar en ellas, así como su significado. El cuestionario fue remitido posteriormente por cada autor a los individuos identificados por ellos como los mejores conocedores del tema en estudio. Los datos fueron recogidos en el año 2002.

Las actividades sanitarias identificadas, representando los tres aspectos señalados de la provisión de servicios sanitarios, fueron ocho: dos en prevención primaria (vacunaciones infantiles y vacunación antigripal de adultos); seis en prevención secundaria (detección precoz de: cáncer de mama mediante mamografía, tuberculosis, Hipertensión Arterial (HTA), fenilcetonuria (PKU), virus de la inmunodeficiencia humana (HIV), y osteoporosis), y seis de prevención terciaria (relativas al manejo clínico y seguimiento de los diagnósticos positivos en las seis actividades de prevención secundaria).

Para cada una de las actuaciones clínicas definidas se trataba de conocer la población diana: por edad y sexo en función de la intervención, poblaciones seleccionadas por tener un riesgo particularmente alto (por ejemplo personas que viven en un área geográfica concreta con exposiciones elevadas), o individuos concretos en riesgo elevado (por ejemplo aquéllos con antecedentes personales o familiares relacionados con las enfermedades estudiadas). Además, se buscaba conocer el nivel en el que se ubicaba la responsabilidad para la toma de decisiones en relación con diferentes aspectos del proceso de atención (en una Autoridad administrativa, en la Atención Primaria, en el Hospital, o en organizaciones profesionales):

1. Quién define la política en relación con la organización del proceso de atención y dónde se provee.
2. Quién efectúa el contacto con los individuos para poder realizar la actuación.

3. Quién realiza el seguimiento de los individuos con resultados patológicos.

4. Quién mantiene los registros clínicos de los resultados obtenidos y los procedimientos subsiguientes. Este aspecto del proceso de atención entra dentro del manejo clínico y es susceptible para todas las actividades preventivas excepto aquellas de prevención primaria (vacunaciones).

Para la realización del estudio se eligieron ocho áreas en seis países diferentes: Bélgica; Québec (Canadá); Estado de Illinois (EEUU); Andalucía (España); Asturias (España); Madrid (España); Noruega; y Reino Unido. Las áreas fueron seleccionadas por tener diferentes modelos de sistemas sanitarios y por diferir también en su orientación hacia la atención primaria ${ }^{10}$. Reino Unido, España y Noruega son países con una infraestructura en Atención Primaria importante. En España, las Comunidades Autónomas tienen capacidad para organizar sus Servicios de Salud, por lo que se eligieron tres de ellas, una con competencias plenas desde 1984 (Andalucía) y las otras dos desde 2002 (Asturias y Madrid). En Canadá las provincias están en un nivel intermedio en su orientación hacia la atención primaria, mientras que EEUU y Bélgica son países con un desarrollo débil de la atención primaria.

\section{RESULTADOS}

Las tablas 1 a 8 recogen los resultados encontrados para cada actividad preventiva, los cuales se resumen en esta sección agrupados por las variables de estudio analizadas.

A Poblaciones diana. En las ocho áreas geográficas estudiadas la población diana 
Tabla 1

Hallazgos en relación con las INMUNIZACIONES INFANTILES

\begin{tabular}{|c|c|c|c|c|}
\hline & País & $\begin{array}{c}\text { Población } \\
\text { diana }\end{array}$ & Establecimiento de políticas & $\begin{array}{c}\text { Lugar de contacto con } \\
\text { los individuos } \\
\end{array}$ \\
\hline \multicolumn{2}{|l|}{ Bélgica } & Toda & Autoridad Administrativa & Atención Primaria \\
\hline \multicolumn{2}{|c|}{ Canadá, Quebec } & Toda & Autoridad Administrativa & Variable \\
\hline \multirow{3}{*}{ España } & Andalucía & Toda & Autoridad Administrativa & Atención Primaria \\
\hline & Asturias & Toda & Autoridad Administrativa & Atención Primaria \\
\hline & Madrid & Toda & Autoridad Administrativa & Atención Primaria \\
\hline \multicolumn{2}{|c|}{ EEUU, Illinois } & Toda & Autoridad Administrativa & Atención Primaria \\
\hline \multicolumn{2}{|c|}{ Noruega } & Toda & Autoridad Administrativa & Atención Primaria \\
\hline \multicolumn{2}{|c|}{ Reino Unido } & Toda & Autoridad Administrativa & Atención Primaria \\
\hline
\end{tabular}

Toda: toda la población definida como susceptible para la intervención por edad o sexo.

Tabla 2

Hallazgos en relación con INMUNIZACIÓN GRIPE

\begin{tabular}{|c|c|c|c|c|}
\hline \multicolumn{2}{|c|}{ País } & Población diana & Establecimiento de políticas & $\begin{array}{c}\text { Lugar de contacto con } \\
\text { los individuos }\end{array}$ \\
\hline \multicolumn{2}{|l|}{ Bélgica } & Toda & Grupo profesional & Atención Primaria \\
\hline \multicolumn{2}{|c|}{ Canadá, Quebec } & Grupo de riesgo & Autoridad Administrativa & Variable \\
\hline \multirow{3}{*}{ España } & Andalucía & Toda & Autoridad Administrativa & Atención Primaria \\
\hline & Asturias & Toda & Autoridad Administrativa & Atención Primaria \\
\hline & Madrid & Toda & Autoridad Administrativa & Atención Primaria \\
\hline \multicolumn{2}{|c|}{ EEUU, Illinois } & Toda & Autoridad Administrativa & Atención Primaria \\
\hline \multicolumn{2}{|c|}{ Noruega } & Individuo & Autoridad Administrativa & Atención Primaria \\
\hline \multicolumn{2}{|c|}{ Reino Unido } & Toda & Autoridad Administrativa & Atención Primaria \\
\hline
\end{tabular}

Toda: toda la población definida como susceptible para la intervención por edad o sexo. Grupo de riesgo: Población seleccionada por tener un riesgo potencialmente alto.

Individuo: personas concretas con riesgo elevado.

era toda la población susceptible por edad o genero para las vacunaciones infantiles, la detección de PKU y la detección precoz de cáncer de mama mediante mamografía. En el resto de las actividades preventivas la población diana variaba en las diferentes áreas estudiadas, pudiendo ser toda la población susceptible, grupos de riesgo, o individuos.

B. Establecimiento de las políticas de prevención. Sólo para tres actividades preventivas el nivel de responsabilidad era una autoridad administrativa en las ocho áreas: vacunaciones infantiles, detección PKU y detección de cáncer de mama mediante mamografía. En el caso de la tuberculosis, la responsabilidad se localizaba en una autoridad administrativa en siete de las áreas, y en la octava (Andalucía) en los Hospitales. En el resto de las actividades preventivas variaba entre grupos profesionales, unidades administrativas, en el hospital o era variable.

C. Lugar de contacto con los individuos de la población diana en función de la actividad de prevención. Había poca homogeneidad entre las áreas, excepto en las actividades de prevención primaria, donde la responsabilidad era asumida por los centros de atención primaria en siete de ellas, mientras que en Québec la responsabilidad estaba difuminada entre atención primaria y atención hospitalaria. Para la HTA también se 
Tabla 3

Hallazgos en relación con la detección con de cáncer de mama

\begin{tabular}{|c|c|c|c|c|c|}
\hline País & $\begin{array}{c}\text { Población } \\
\text { diana }\end{array}$ & Establecimiento de políticas & $\begin{array}{c}\text { Lugar de contacto con los } \\
\text { individuos }\end{array}$ & $\begin{array}{c}\text { Lugar del } \\
\text { seguimiento }\end{array}$ & $\begin{array}{l}\text { Mantenimiento } \\
\text { registros Clínicos }\end{array}$ \\
\hline Bélgica & Toda & Autoridad Administrativa & Autoridad Administrativa & Variable & Atención Primaria \\
\hline Canadá Quebec & Toda & Autoridad Administrativa & Autoridad administrativa & Variable & Variable \\
\hline I Andalucía & Toda & Autoridad Administrativa & Hospital & Hospital & Hospital \\
\hline Asturias & Toda & Autoridad Administrativa & Variable & Hospital & Hospital \\
\hline Madrid & Toda & Autoridad Administrativa & Atención Primaria & Hospital & Atención Primaria \\
\hline EEUU, Illinois & Toda & Autoridad Administrativa & Atención Primaria & $\begin{array}{l}\text { Atención } \\
\text { Primaria }\end{array}$ & Variable \\
\hline Noruega & Toda & Autoridad Administrativa & Autoridad Administrativa & $\begin{array}{l}\text { Atención } \\
\text { Primaria }\end{array}$ & Atención Primaria \\
\hline Reino Unido & Toda & Autoridad Administrativa & Variable & Hospital & Hospital \\
\hline
\end{tabular}

Toda: toda la población definida como susceptible para la intervención por edad o sexo.

Tabla 4

Hallazgos en relación con la detección de TUBERCULOSIS

\begin{tabular}{|c|c|c|c|c|c|}
\hline País & Población diana & $\begin{array}{c}\text { Establecimiento de } \\
\text { políticas }\end{array}$ & $\begin{array}{c}\text { Lugar de contacto con los } \\
\text { individuos }\end{array}$ & $\begin{array}{c}\text { Lugar del } \\
\text { seguimiento }\end{array}$ & $\begin{array}{c}\text { Mantenimiento registros } \\
\text { clínicos }\end{array}$ \\
\hline Bélgica & Individuo & Autoridad Administrativa & Atención Primaria & Variable & Atención Primaria \\
\hline Canadá Quebec & Grupo de riesgo & Autoridad Administrativa & Variable & Variable & Variable \\
\hline I Andalucía & Individuo & Hospital & Hospital & Hospital & Hospital \\
\hline Asturias & Individuo & Autoridad Administrativa & Variable & Variable & Variable \\
\hline की Madrid & Individuo & Autoridad Administrativa & Atención primaria & Variable & Atención Primaria \\
\hline EEUU, Illinois & Grupo de riesgo & Autoridad Administrativa & Atención Primaria & Variable & Atención Primaria \\
\hline Noruega & Toda & Autoridad Administrativa & Autoridad administrativa & Hospital & Autoridad administrativa \\
\hline Reino Unido & Individuo & Autoridad Administrativa & Variable & Atención Primaria & Atención Primaria \\
\hline
\end{tabular}

Toda: toda la población definida como susceptible para la intervención por edad o sexo.

Grupo de riesgo: Población seleccionada por tener un riesgo potencialmente alto.

Individuo: personas concretas con riesgo elevado.

Tabla 5

Hallazgos en relación con la detección HTA

\begin{tabular}{|c|c|c|c|c|c|}
\hline País & Población diana & $\begin{array}{l}\text { Establecimiento de } \\
\text { políticas }\end{array}$ & $\begin{array}{l}\text { Lugar de contacto } \\
\text { con los individuos }\end{array}$ & $\begin{array}{c}\text { Lugar del } \\
\text { seguimiento }\end{array}$ & $\begin{array}{c}\text { Mantenimiento } \\
\text { registros clínicos }\end{array}$ \\
\hline Bélgica & Individuo & Variable & Variable & Atención Primaria & Atención Primaria \\
\hline Canadá Quebec & Individuo & Grupo profesional & Variable & Atención Primaria & Variable \\
\hline I Andalucía & Grupo de riesgo & Autoridad administrativa & Atención Primaria & Atención Primaria & Atención Primaria \\
\hline Asturias & Grupo de riesgo & Autoridad administrativa & Atención Primaria & Atención Primaria & Atención Primaria \\
\hline If Madrid & Toda & Autoridad administrativa & Atención Primaria & Atención Primaria & Atención Primaria \\
\hline EEUU, Illinois & Toda & Grupo profesional & Atención Primaria & Atención Primaria & Atención Primaria \\
\hline Noruega & Individuo & Grupo profesional & Atención Primaria & Atención Primaria & Atención Primaria \\
\hline Reino Unido & Toda & Autoridad Administrativa & Atención Primaria & Atención Primaria & Atención Primaria \\
\hline
\end{tabular}

Toda: toda la población definida como susceptible para la intervención por edad o sexo.

Grupo de riesgo: Población seleccionada por tener un riesgo potencialmente alto.

Individuo: personas concretas con riesgo elevado.

contactaba en atención primaria, excepto en Bélgica y en Canadá. En el resto de las actividades preventivas el lugar de contacto se hacía bien en el hospital o era variable.
D. Lugar de responsabilidad para el seguimiento de los diagnósticos positivos detectados por las actuaciones de prevención secundaria. Ningún área identificó una 
Tabla 6

Hallazgos en relación con la detección PKU

\begin{tabular}{|lccccc|}
\hline \multicolumn{1}{|c}{ País } & $\begin{array}{c}\text { Población } \\
\text { diana }\end{array}$ & Establecimiento de políticas & $\begin{array}{c}\text { Lugar de contacto con los } \\
\text { individuos }\end{array}$ & $\begin{array}{c}\text { Lugar del } \\
\text { seguimiento }\end{array}$ & $\begin{array}{c}\text { Mantenimiento } \\
\text { registros clínicos }\end{array}$ \\
\hline Bélgica & Toda & Autoridad Administrativa & Autoridad Administrativa & Hospital & Atención Primaria \\
Canadá Quebec & Toda & Autoridad Administrativa & Hospital & Hospital & Hospital \\
Andalucía & Toda & Autoridad Administrativa & Autoridad Administrativa & Hospital & Hospital \\
And Asturias & Toda & Autoridad Administrativa & Atención Primaria & Hospital & Hospital \\
W Madrid & Toda & Autoridad Administrativa & Hospital & Hospital & Atención Primaria \\
EEUU, Illinois & Toda & Autoridad Administrativa & Hospital & Variable & Variable \\
Noruega & Toda & Autoridad Administrativa & Atención Primaria & Hospital & Atención Primaria \\
Reino Unido & Toda & Autoridad Administrativa & Variable & Hospital & Hospital \\
\hline
\end{tabular}

Toda: toda la población definida como susceptible para la intervención por edad o sexo.

Tabla 7

Hallazgos en relación con la detección HIV

\begin{tabular}{|c|c|c|c|c|c|}
\hline País & Población diana & $\begin{array}{c}\text { Establecimiento de } \\
\text { políticas }\end{array}$ & $\begin{array}{l}\text { Lugar de contacto } \\
\text { con los individuos }\end{array}$ & $\begin{array}{c}\text { Lugar del } \\
\text { seguimiento }\end{array}$ & $\begin{array}{l}\text { Mantenimiento } \\
\text { registros clínicos }\end{array}$ \\
\hline Bélgica & Individuo & Variable & Variable & Variable & Atención Primaria \\
\hline Canadá Quebec & Grupo de riesgo & Autoridad Administrativa & Variable & Variable & Variable \\
\hline Andalucía & Individuo & Hospital & Hospital & Hospital & Hospital \\
\hline Asturias & Individuo & Hospital & Hospital & Hospital & Hospital \\
\hline II Madrid & Individuo & Autoridad Administrativa & Atención Primaria & Hospital & Atención Primaria \\
\hline EEUU, Illinois & Individuo & Autoridad Administrativa & Atención Primaria & Variable & Atención Primaria \\
\hline Noruega & Individuo & Autoridad Administrativa & Atención Primaria & Hospital & Hospital \\
\hline Reino Unido & Grupo de riesgo & Autoridad Administrativa & Variable & Hospital & Variable \\
\hline
\end{tabular}

Grupo de riesgo: Población seleccionada por tener un riesgo potencialmente alto.

Individuo: personas concretas con riesgo elevado.

Tabla 8

Hallazgos en relación con la detección OSTEOPOROSIS

\begin{tabular}{|c|c|c|c|c|c|}
\hline País & $\begin{array}{c}\text { Población } \\
\text { diana }\end{array}$ & $\begin{array}{c}\text { Establecimiento de } \\
\text { políticas }\end{array}$ & $\begin{array}{l}\text { Lugar de contacto } \\
\text { con los individuos }\end{array}$ & $\begin{array}{c}\text { Lugar del } \\
\text { seguimiento }\end{array}$ & $\begin{array}{c}\text { Mantenimiento } \\
\text { registros clínicos }\end{array}$ \\
\hline Bélgica & Individuo & Variable & Variable & Variable & Atención Primaria \\
\hline Canadá Ouebec & Individuo & Grupo profesional & Variable & Variable & Variable \\
\hline Andalucía & Toda & Hospital & Atención Primaria & Atención Primaria & Atención Primaria \\
\hline$\cong$ Asturias & Toda & Hospital & Variable & Variable & Atención Primaria \\
\hline If Madrid & Individuo & Autoridad Administrativa & Atención Primaria & Hospital & Atención Primaria \\
\hline EEUU, Illinois & Toda & Grupo profesional & Atención Primaria & Atención Primaria & Variable \\
\hline Noruega & Individuo & Hospital & Atención Primaria & Atención Primaria & Atención Primaria \\
\hline Reino Unido & Individuo & Variable & Variable & Variable & Variable \\
\hline
\end{tabular}

Toda: toda la población definida como susceptible para la intervención por edad o sexo.

Individuo: personas concretas con riesgo elevado.

autoridad o unidad administrativa como responsable para el seguimiento de las diferentes situaciones clínicas estudiadas. La atención primaria realizaba el seguimiento del cáncer de mama en EEUU y Noruega, de la tuberculosis en el Reino Unido, en todas las áreas geográficas estudiadas para el caso de la HTA, y en Andalucía, EEUU y Noruega para la osteoporosis. La atención primaria en ningún caso intervenía en el seguimiento para los diagnósticos de PKU. El hospital es el nivel más frecuente para el seguimiento en 
el caso de detección de personas HIV positivas, PKU positivas, pero, llamativamente, sólo en cuatro casos el hospital realizaba el seguimiento de las mujeres diagnosticadas de cáncer. Esto se hacía así en las tres áreas estudiadas en España y en el reino Unido.

E. El lugar de responsabilidad para el mantenimiento de los registros clínicos era igualmente variable entre las ocho áreas. En ningún caso el lugar era una autoridad o unidad administrativa. La atención primaria era citada con más frecuencia como la responsable de esta función en el caso de HTA (en siete áreas excepto en Canadá), y de la osteoporosis (en cinco áreas). En el resto de las actividades preventivas el mantenimiento de los registros en las diferentes áreas se llevaban por el hospital, por una autoridad administrativa o era variable.

\section{DISCUSIÓN}

Este estudio piloto sobre dónde se ubica la responsabilidad en diferentes aspectos de atención para los procesos preventivos seleccionados mostró muy pocas coincidencias tanto dentro de las diferentes áreas estudiadas como entre ellas, sugiriendo que el tema de donde debería ubicarse de forma más apropiada el nivel de responsabilidad está pendiente de deliberación dentro de cada país y entre ellos. Parece que hay un consenso limitado o una evidencia limitada con relación a la mejor manera de realizar las intervenciones preventivas que conllevan prestación de servicios sanitarios individualizados. El estudio muestra también la gran heterogeneidad existente entre las tres áreas analizadas en España a pesar del esfuerzo realizado tanto por las sociedades científicas y las administraciones ${ }^{11-13}$ como con la introducción de instrumentos de gestión tales como el contrato programa ${ }^{14}$, que incluye una amplia cartera de servicios y normas técnicas mínimas en Atención Primaria.

El estudio tiene diversas limitaciones que deben ser mencionadas. En primer lugar, los hallazgos están basados en informes de personas expertas; aunque éstas fueron seleccionadas sobre la base de su conocimiento del área en la que trabajan, no puede asegurarse que todas las respuestas reflejen perfectamente el estado actual de los diferentes temas. Sin embargo, el hecho de que las respuestas fuesen tan variables para las diferentes actividades de prevención y entre las distintas áreas, sugiere que los informadores no respondieron de forma general, sino más bien que consideraron cada respuesta independientemente.

En segundo lugar, los hallazgos son meramente descriptivos y no se pretende relacionar las diferencias con aspectos concretos de los sistemas sanitarios así como tampoco buscar las razones históricas para las diferencias. En algún momento será necesario entender las bases para las diferencias en las diversas actividades de prevención entre áreas o en un mismo país, y si ello tiene algún impacto en relación con la cobertura de los diferentes servicios.

Debido a la ausencia de cualquier consistencia en relación con los diferentes sistemas sanitarios, parece poco probable que las líneas de responsabilidad para los diferentes tipos de servicios sanitarios personales estén mejor delimitadas por el grado de orientación de los sistemas sanitarios hacia poblaciones concretas. Wagner and LoGerfo ${ }^{16}$ han señalado que tanto la medicina como la salud pública han integrado cada una las actividades de la otra: Independientemente de su legitimidad histórica, la distinción [entre salud pública y medicina] representa de forma inadecuada la salud pública y la práctica clínica contemporáneas.

Rundall sugiere cinco condiciones para una integración con éxito de la salud pública y la medicina: una visión compartida, un mandato claro, una inversión mayor en promoción de la salud colectiva y en la prevención de la enfermedad en las actividades de salud pública, una mayor inversión en pre- 
vención clínica y atención primaria, y un «espíritu empresarial» en el sector sanitario público $^{17}$. Los críticos con su artículo ${ }^{18-20}$ señalan algunas discrepancias con la conceptualización de las áreas que se superponen y aunque parece haber una considerable convergencia en la idea de que las funciones de salud pública podrían ser complementarias a aquéllas del sistema médico de cuidados en general, y más en concreto de la atención primaria, hay escaso acuerdo en cómo deberían implementarse. Si las funciones de salud pública de evaluación, desarrollo de políticas y garantías, se trasladan a actividades, incluyendo la documentación de las necesidades de salud, el establecimiento de estándares (objetivos) para responder a esas necesidades, la monitorización del cumplimiento de los objetivos, y la regulación de las actividades para reestablecer metas y las acciones precisas para responder a las necesidades, todavía se plantean dudas en relación con la ejecución de la determinación de las necesidades, cómo se realiza el establecimiento de estándares y cómo se hace aceptable y efectiva la regulación.

Las experiencias de otros países industrializados pueden suministrar información que podría ayudar a delimitar las posibles alternativas para afrontar estos retos. Para ello debe tenerse en cuenta que los diferentes países difieren ampliamente en la intensidad del control público de sus sistemas sanitarios. Como Kirkman-Liff ${ }^{21}$ señala, esta variación va desde sistemas sanitarios (incluyendo la salud pública) dirigidos por los gobiernos, seguros sociales administrados por los gobiernos, seguros mixtos, y seguros privados. Además, los diferentes países difieren en su compromiso con la atención primaria como base para sus sistemas sanitarios y, por tanto, es posible que exista una amplia variedad de aproximaciones que sobrepasan las fronteras de lo público/privado y de la población/paciente. De hecho, los países mantienen diferencias en la extensión con la que sus sistemas sanitarios se orientan en torno a una base de atención primaria fuerte. La fuerza de esta base puede cuantificarse y sus diferentes componentes pueden ser evaluados ${ }^{15}$.

El interés en la aplicación potencial de experiencias de otros países es aún mayor como consecuencia de que la mayoría de los países europeos, incluyendo España y sus Comunidades Autónomas, están llevando a cabo reformas en sus sistemas sanitarios ${ }^{22-24}$, introduciendo cierto grado de privatización de los servicios, con el objeto prioritario de reducir el importante crecimiento de los costes sanitarios, al tiempo que en Europa se pretende preservar las características básicas de sus sistemas sanitarios que consisten, aún con una cierta variabilidad, en un compromiso con la universalidad de las prestaciones y el principio de $«$ solidaridad ${ }^{25}$.

Mientras la racionalidad subyacente para considerar algo dentro de la esfera de la salud pública no sea mejor entendida, es muy probable que continúe la considerable variación en estos procesos de atención o, quizás, incluso que aumente al ritmo en que se incremente el rango de acciones de intervención. La importancia de estas diferencias para la efectividad de los cuidados merece atención.

\section{BIBLIOGRAFÍA}

1. Múzquiz Vicente-Arche R. Análisis del proceso de transferencias sanitarias. Revista de Administración Sanitaria 2000; IV (16): 603-16.

2. Martín Moreno JM. Hacia un modelo de cooperación y armonización en el campo de la salud pública en España. Rev Esp Salud Pública 2002; 76 : 637-43.

3. Holland WW, Detels R, Knox G. Oxford Textbook of Public Health, 2nd edition. Volume 1: Influences of Public Health. Oxford, UK, and New York, NY: Oxford University Press; 1991.

4. Institute of Medicine. The Future of Public Health. Washington, DC: National Academy Press; 1988.

5. World Health Organisation. Primary Health Care. Geneva: World Health Organisation; 1978 (Health for All Series $N^{0} 1$ ). 
6. Marset Campos P. Estructuras Político-Administrativas y Salud Pública en España. Rev Sanid Hig Pública 1994; 68: 57-64.

7. Martín Zurro A. Prevención y promoción de la salud en Atención Primaria. Rev Sanid Hig Pública 1991; 66: 477-79.

8. Gillam S, Abbott S, Banks-Smith J. Can primary care groups and trusts improve health? BMJ 2001; 323(7304):89-92.

9. Starfield B. Public health and primary care: a framework for proposed linkages. Am J Public Health 1996; 86(10):1365-69.

10. Starfield B, Shi L. Policy relevant determinants of health: an international perspective. Health Policy 2002; 60(3):201-18

11. Ascunze Elizaga N, González Enríquez J, González Navarro A., Herranz Fernández C, Marqués Bravo A, y Martín Pérez J. Criterios generales y recomendaciones para la elaboración de programas de detección precoz de cancer de mama y cancer de cerviz uterino en España. Rev Sanid Hig Pública 1993; 67: 23-37.

12. Villar F, Banegas JR, Gil E y Aranda P. Por un mejor control de la hipertensión arterial en España. Rev Esp Salud Pública 1996; 70: 134-138

13. Diez Ruiz-Navarro M. La tuberculosis en los albores del siglo XXI. Rev Esp Salud Pública 2003; 77: 183-187

14. Pérez Andrés C. Aceptación del Contrato Programa por el personal sanitario de la Atención Primaria en Madrid: un estudio cualitativo. Rev Sanid Hig Pública 1995; 69: 79-88.

15. Starfield B. Primary Care: Balancing Health Needs, Services, and Technology. New York: Oxford University Press; 1998.
16. Wagner E, LoGerfo J. The Medical-Public Health Bridge: Integrating and Financing Clinical Prevention. Rebalancing the Triad: Cure, Care, and Prevention. Sun Valley, ID: Sun Valley Forum; 1992.

17. Rundall TG. The integration of public health and medicine. Front Health Serv Manage 1994; 10(4):3-24.

18. Burdine JN, Felix MR. Beyond the integration of public health and medicine. Front Health Serv Manage 1994; 10(4):26-31.

19. Roper WL, Koplan JP, Stinnett AA. Public health in the new American health system. Front Health Serv Manage 1994; 10(4):32-36.

20. Van Amringe M. A national architecture for integration. Front Health Serv Manage 1994; 10(4):3741.

21. Kirkman-Liff BL. Management without frontiers: health system convergence leads to health care management convergence. Front Health Serv Manage 1994; 11(1):3-48.

22. OECD. New Directions in Health Care Policy. Health Policy Studies No. 7. Paris: OECD; 1995.

23. Saltman RB, Figueras J. European Health Care Reform: Analysis of Current Strategies. Copenhagen: WHO Regional Office for Europe;1997.

24. OECD. Measuring Up: improving health system operformance in OECD countries. Paris: OECD; 2002.

25. Government Committee on Choices in Health Care. Choices in Health Care. Amsterdam: Ministry of Welfare, Health, and Cultural Affairs; 1992. 